\title{
In regulation we trust
}

\author{
Siri Wiig ${ }^{\mathrm{a}, b^{*}}$, Jorunn Elise Tharaldsen ${ }^{\mathrm{b}}$ \\ ${ }^{a}$ University of Stavanger, N-4036 Stavanger, Norway \\ ${ }^{\mathrm{b}}$ Petroleum Safety Authority Norway, Professor Olav Hansens vei 10, NO-4003 Stavanger, Norway
}

\begin{abstract}
The role of trust has been argued to play an increasingly important role in modern, complex, and ambivalent risk societies. Trust within organizational research is anticipated to have a general strategic impact on aspects such as organizational performance, communication and knowledge exchange, and learning from accidents. Trust is also an important aspect related to regulation of risk. Diverse regulatory regimes, their contexts and risks influence regulators use of trust and distrust in regulatory practice. The aim of this paper is to discuss the relationship between risk regulation and trust across diverse risk regulation regimes. By drawing from studies of risk regulation, risk perception, and trust the purpose is to discuss how regulation and trust are linked and used in practice to control risk across system levels in socio-technical systems in high risk industries. This paper provides new knowledge on 1) how functional and dysfunctional trust and distrust are grounded in the empirical realities of high risk industries, 2) how different perspectives on trust and distrust act together and bring new knowledge on how society control risk.
\end{abstract}

Keywords: trust, distrust, risk, regulation

*Corresponding author. E-mail: siri.wiig@uis.no or siri.wiig@ptil.no, telephone: 0047-51834288, fax: 0047-51834150. 


\section{Introduction}

The role of trust has been argued to play an important role in modern, global, complex, and ambivalent risk societies [1-3] and to be a cornerstone in the creation of social order [4]. The trust concept is also regarded to be a complex, multidisciplinary, multifaceted, multilevel and multiplex phenomena $[4,5]$. Despite differing conceptualizations, there seems to be some agreement on the meaning of trust, the conditions that must exist for trust to arise and its functions. It implies positive expectations about others' intentions and behavior, it reduces complexity and conflict, involves vulnerability and risk, and it involves interdependence between different types of actors. Research on trust has commonly been related to positive organizational outcomes, like increased competitiveness [6], higher organizational performance [7], reduced transaction costs [8] increased communication and knowledge exchange [9], enhanced mutual learning [10], learning from accidents [11], and is seen to have a positive impact on safety culture and safety performance $[11,12]$. Diverse regulatory regimes, their contexts and risks [13] influence regulators use of trust and distrust in regulatory practice. The aim of this paper is to discuss the relationship between risk regulation and trust across diverse risk regulation regimes. By drawing from our studies of risk regulation, risk perception, and trust the purpose is to discuss how regulation and trust are linked and used in practice to control risk across system levels in socio-technical systems in high risk industries. This paper should provide new knowledge on 1) how functional and dysfunctional trust and distrust might be grounded in the empirical realities of high risk industries, 2) how different perspectives on trust and distrust may act together and bring new knowledge on how society control risk.

\section{Theoretical approach}

\subsection{Trust}

Most of the research on trust and distrust relies on the assumption that trust on different levels is beneficial and that lack of trust is bad. Lately this view has been challenged, and there now seem to be new perspectives emerging that place emphasis on the limits of trust and the potential benefits of distrust [11,1416]. One might have too much trust ending in naivety or blindness, while too high a level of distrust, for instance in the form of too much monitoring and con- trol might result in a non-sharing environment, inefficiency or other non-intended side-effects. The most common trust promoting factors has been linked to ability, integrity and benevolence [17]. Ability refers to the trustee's skills and competencies, while benevolence is connected to the extent to which a trustee is believed to want to do good to the trustor. Integrity relates to the trustor's perception that the trustee adheres to a set of principles that the trustor finds acceptable, moral integrity, the consistency of the party's actions, credible communications, justice etc [17].

The influence of culture on trust building processes has been touched upon and, despite differences in focus, all studies assert that trust or institutional elements facilitating trust have a positive influence on various organizational aspects, and that the level or type of trust differs between cultures $[18,19]$.

Our understanding of trust relies on an assumption that both trust and distrust may be functional as well as dysfunctional - the overall aim with both is to create predictability. This is also in accordance with a Luhmanian perspective on trust, which claims that both a trusting and distrusting strategy involves reduction of complexity, i.e. trust reduces social complexity by allowing specific undesirable conduct to be removed from consideration, while distrust functions to reduce complexity by allowing undesirable conduct to be seen as likely - even certain [4]. This perspective on trust implies that trust and distrust may have both a functional and dysfunctional impact on safety and effective risk regulation. Dysfunctional trust and dysfunctional distrust are hypothesized to give poor safety performance, while functional trust and functional distrust should lead to sound safety performance.

\subsection{The control of risk}

How regulators approach their role and function appears to be culturally dependent. Similar regulatory challenges result in different regulatory solutions in different regimes and different nations. The way regulators conceive their mission and their regulatees is important in explaining the approaches of different regimes [20-22]. A vital aspect in understanding risk regulation processes is to study regulatory enforcement practices. Just as it is important to know how standards are formed, it is also important to gain knowledge about how these are transmitted downward the regime levels or sub-systems, and implemented at the street level. The complexity of regula- 
tory regimes, the complexity of problems in the area to be regulated, and the distance between regulatory authority and the regulated, all make the issue of how to control the discretion at the street level crucial [21].

It is difficult for risk regulators to choose the appropriate enforcement strategies to target the optimal method of regulating risks. Regulators seek to enforce compliance with the law, not merely through formal enforcement and prosecution, but also through a host of informal techniques, including education, advice, persuasion, and negotiation [23]. According to Kagan and Scholz (1984), problems of regulatory enforcement usually refer to the motives, attitudes, and capabilities of the regulatee. Three "images" of the regulatee are created in the literature with corresponding theories to explain non-compliance. The first image depicts the regulatee as amoral calculator, motivated entirely by profit. This regulatee disobeys the law if it is beneficial to do so, and noncompliance stems from economic calculation. The second image depicts the regulatee as a political citizen, tending to comply with the law, partly because of a belief in the law, and partly because of long-term interest. In this case, non-compliance stems from principled disagreements with regulations that are sometimes regarded as arbitrary or unreasonable. The third image depicts the regulatee as organizationally incompetent, whose intentions are to obey the law, however it is potentially fallible due to lack of organizational capacity to do so. In this case the noncompliance arises from organizational failure. Each of these images requires different regulatory enforcement strategies $[20,24]$ and roles for the regulators $[11,25]$. To deal with the amoral calculators the regulatory authority should emphasize aggressive inspection or with a functional distrusting approach. The goal is deterrence and the inspectors appear as policemen. To deal with the political citizen the regulatory authority should act as a politician, persuading the regulated of the rationality of the case; a role which to a larger extent emphasizes a functional trusting attitude towards the regulatee. In order to deal with the organizationally incompetent entity the regulator should serve as a consultant, bridging the competence gap through education. In this third strategy both a functional trusting and distrusting strategy may be used. Each of these theories of corporate legal behavior or misbehavior, capture important aspects of reality. However, the diverse sources of non-compliance imply that reliance on any single theory of non-compliance is likely to be wrong, and, when translated into enforcement strategies, counteractive. This means that regulators need to be adap- tive and should be aware that non-compliance may have multiple reasons. Regulatory inspectors must be prepared to shift roles according to their analysis of the regulated organization [22,24-26].

These theoretical contributions illustrate that trust, distrust and control are vital aspects of how regulators approach the regulatees. In practice, diverse regimes should use diverse combinations of trust, distrust and control, depending on the context and type of regulatee the regulator are approaching.

\section{Methodology}

\subsection{Research strategy}

This paper has a macro ergonomic perspective and examine risk regulation, trust and distrust in three contrasting sectors in Norway. The paper is based on a multiple case study of the following Norwegian risk regulation regimes: specialized healthcare services, municipal emergency management, and petroleum industry. The paper applies a dissimilar case study approach to generate contrasting data to explore regime context, content and the function of trust and distrust within the regimes. The cases have been selected due to their diversity in instrumental and institutional aspects, such as type and scale of hazard, diverse formal rule specification, sanction authority, history, enforcement strategies, tools and practices used by the state. Such different cases allow the study of regimes with diverse balances between the regime content and regime context, thereby enhancing the analysis of the role of trust and distrust across regimes as well as mapping social and institutional factors affecting trust [27].

\subsection{Data collection}

This study makes use of both new data and data gathered and analyzed in former studies 1) [28], 2) [29] and 3) [27]. The two first studies are based on both qualitative and quantitative methods collected in the petroleum sector. The first study deals with the functions of trust and distrust and their relations to safety within a group of drilling and well service contractors on the Norwegian and the UK Continental Shelves, and is based on quantitative and qualitative data. The second study compares four major petroleum accidents and their effects on risk regulation. It is based on qualitative data; mainly document analysis of former accident investigation reports, and other 
available material such as film documentaries, research reports and literature.

The third study used a combined methods approach of qualitative and quantitative data such as interviews, document analyses, observations, and statistical analyses [30]. A total of forty-nine taperecorded interviews were performed, at different system levels within the specialized healthcare field, using two structured interview guides. Seventeen interviews dealt with the relationship between the local health regulator and the regulatee (hospital) with regards to error management using interview guide A (legislation, error reporting, learning, risk perception, and prevention). Informants included inspectors, the patient representative association, and hospital management. Thirty-two interviews examined how two hospital divisions handled errors using interview guide B (amount and categorization, human and organizational factors, learning, power issues, and regulators role) [31].

Within municipal emergency management, the study examined six municipalities along with the regulatory authority in two counties. The municipality cases varied with regards to population, size, location, risk sources, size of emergency management staffs, occurrences of severe accidents, and how regulators evaluated the emergency plans and exercises. Twelve tape-recorded interviews were conducted with municipal emergency management employees and regulatory inspectors, following an interview guide that included questions related to risk perception, risk communication, learning, and trust. These issues were included in order to conduct a broad exploration of how risks are identified and communicated by regulatory inspectors and municipal emergency managers, how municipalities apply risk information for learning purposes, and how the aspect of trust influences the activities between risk regulator and municipalities.

\subsection{Data analysis}

In this paper we have merged our data from the different case studies and analyzed how trust, distrust and control are used in different contexts. We have focused on enforcement strategies, competence, contextual differences, and risk profiles.

\section{Results}

\subsection{Context and risk profiles}

Our results illustrate contrasting contexts and risk sources to be controlled. The society responds to different risks by developing different risk regulation regimes. Within the specialized healthcare, the medical error frequency is high, causing approximately 2000 patient deaths a year in Norwegian hospitals [32]. However, the potential for catastrophe is low because medical errors harm one patient at a time (excluding epidemics and fires). In municipal emergency management, the risk profile is characterized with various hazards; some represent a significant potential for catastrophe but have a low occurrence probability (e.g breakdown in infrastructure such as water supply), while others have a higher occurrence probability, but does not involve a high catastrophic potential (e.g avalanche). The petroleum industry is characterized by risks having a higher catastrophic potential, but lower probability.

The diversity in risk profiles generate diverse legislative regimes and thus different approaches to trust and distrust in regulatory practice. The regulation within municipal emergency management did not entail a comprehensive legislation and the regime lacked the formal legislative framework and relied to a large degree on governmental expectations and the internal will and acknowledgement among municipalities to take action on risk within the municipalities. The specialized health care regime had developed several legal acts focusing individual health care personnel, health care institutions, patient rights and a systematic approach to quality and safety. The petroleum industry has been in the forefront in Norway in relation to establish a performance based regulatory regime. The legislation is comprehensive and functional and to a strong degree employs a system perspective. Sanctions are usually directed towards systems. We see that the tripartite collaboration between regulator, unions and the industry organizations is vital in the petroleum industry, and trust is an important pillar used by the regulator.

\subsection{Enforced self-regulation}

All three regimes are founded on the basis of internal control, enforced self-regulation, and state control. However there exist large differences with re- 
gards to how the regimes organize this type of regulation and how trust is used in practice.

The legislative versus non-legislative element and competence level among actors within the regimes were vital in understanding the approach to trust or distrust in the regimes. The role of trust in municipal emergency management was vital in the regulators enforcement of a vague regulation. Due to the lack of legal authority, the inspectors adopted regulatory styles emphasizing collaboration, personal communication, education, and persuasion in order to enhance the knowledge level among municipal emergency managers. In other words, the inspectors compensated for the lack of formal rules and regulations with a strong degree of interaction among the regulated municipalities to generate good relationships, trust building, and amplified risk perception, particularly among employees who lacked formal education and experience. The inspectors emphasized learning activities in the interface between the regulator and the regulated and arranged courses, conducted emergency exercises, and informed politicians to increase risk awareness. As such, the earlier mentioned trust promoting aspects related to ability and integrity are used as means to build trust and predictability among the regulate and the regulator in order to facilitate proper risk regulation.

In the health care regime trust in the relation between regulator and regulatee were less emphasized, and less personal interaction between regulatory inspectors and health care personnel were reported. The regulator enforced strict and detailed regulations, involving several legal acts related to the rights and duties of the individual healthcare employees and the hospital organizations. A wide range of sanctioning means for both the individuals and organizations supported the regulator. Thus the regulator did not depend on good relations and trust to foster regulatory compliance; they could impose compliance by the use of sanctions. Informants perceived sanctions as being biased against blaming individuals due to more sanctioning means against individuals compared to the $\mathrm{n}$ systems, stated in the law. National and local level inspectors argued in favour of a system approach in patient safety and medical errors. However, they agreed that the regulator is presently supported with a stronger individual sanctioning repertoire. The results showed a larger degree of distrust between the hospital and regulator compared to the municipal emergency management regime. A positive dialogue was evident between the inspectors and the hospital as well as a low threshold to contact each other; however, the interactions between regulator and regulatee were to a strong degree characterized by formal written information exchange. The regulatory practice emphasized control-based activities while advice, education, and persuasion aspects were not predominant in this regime.

During the last 25 years, the regulatory regime within the petroleum industry has changed its approach from a "counterparty" to a "co-player" culture. As such the risk regulation regime is based on selfregulation which implies a goal-setting regime in which legislation is less detailed and more general often in the form of functional requirements. The goal has been to move away from a "compliance mentality" - with a passive duty-holder role - to an active and flexible approach in which the dutyholder's responsibility becomes more explicit [33]. The concept is coined enforced self-regulation (internal control in the Norwegian context) and means that the regulatory process is delegated to stakeholders (license holders), but under conditions given by the regulating authority [16]. The risk regulator within the petroleum industry also saw itself as playing an important role as a trust promoter between different actors and parties within the regime. As such, the Norwegian petroleum context is heavily based on tripartism comprising workforce involvement as a foundation pillar [34]. Trust building through dialogue, empowerment and cooperation between regulator, enterprises and unions are being used as important strategies in the development of a robust risk regulation regime in the Norwegian context. Such trust building strategies are chosen to enable the regulated organizations to perform well rather than to impose requirements that may constrain or limit their performance. The use of functional requirements and industry standards require in itself a high level of trust between regulators and the actors being regulated and means a regulator role which has to balance delicately between a reactive, controlling inspector and a dialogue oriented facilitator towards the industry and the unions [35].

However, we believe that there are vital challenges to be taken into consideration with regards to the limits or the dysfunctional aspects in the performance based regimes. In times of good financial situations innovation and safety improvements are probably easier to foster and promote, than in times of scarce resources. There may also be a potential danger of complacency in the performance based regime, where companies are the holding the competence on how to improve safety level within their organization.

An important finding from the study on drilling contractor employees was related to how trust and 
distrust elements were made important in clientcontractor relations [15]. The qualitative findings suggested that contractor employees sensed a strong element of client control when entering new installations, expressed as an almost tiring need to prove themselves and their abilities. Such institutionalized distrust towards contractors may be especially important in the beginning of a relationship, but is anticipated to loosen up as the trust relation grows in depth and bandwidth [36]. Nomadic workers though, being on the move most of the time, might hardly experience such loosening up. However, this type of distrust might constitute an important risk regulating strategy in the relation between client and supplier creating both functional personal and organizational alertness. As such it might promote a careful navigator attitude among the suppliers, and a functional distrusting attitude from clients, may in fact lead to less accident exposure - although it is experienced as tiring [16].

\section{Discussion and conclusion}

We have seen that the literature distinguish between trust and control and their dynamics in strategic alliances. In our study we found that this was highly relevant within regulatory regimes and interfaces between regulators and regulate, also manifesting itself at the client-supplier level. According to Das \& Teng (1998) confidence in partner relations may create a perceived level of certainty that its associates will pursue mutually compatible interests in the alliance (p. 491). In regulatory interfaces there are no equal partners and even though the risk regulators' main goal is to promote safety, safety is one of several vital goals for the regulated organizations. In our three different regulatory contexts we found different confidence in regulated organizations among the regulators regarding compliance. According to Das \& Teng (1998), confidence comes from two distinct sources: trust and control. Control is treated as a regulating process in order to make the elements of a system more predictable. However, the control mechanisms and level may differ [37]. Among others, Argyris (1952) has suggested that the use of strong control mechanisms imply that one party does not trust the other and might decrease the trust level [38]. While others hold the view that control mechanisms, if used properly, may promote the building of mutual trust [39]. One of Das \& Teng's conclusive remarks is that control mechanisms may either enhance or undermine the trust level, depending on the specific type of control mechanisms partners' use. In this sense, trust will play a moderating role between control mechanisms and control level. In our case studies of municipalities we found that control mechanisms founded on communicative approaches, dialogue, and person - to person interaction where depending on trust and at the same time increased level of trust. Municipal emergency management regime faced structural, cultural, demographic and geographic diversity. However the regime heavily relied on trust to foster compliance among regulatees and to some degree we can characterize several of the municipalities as organizationally incompetent when it comes to knowledge and competence related to emergency management thus implying an enforcement strategy heavily dependent on trust between the regulators and regulated.

In the health care regime we found a regulator less dependent on good relationships, communicative approaches, and persuasion. Although diverse health care institutions vary a lot the we argue that they can be perceived as political citizens with a need for several strategies from the regulator sometimes beyond the most commonly used strategies of system audits. We believe a functional trust approach taking more use of communicative approaches would be positive towards the health care institutions.

In the petroleum industry competence level vary among companies, however the regulator tend to view regulatees as political citizens. The regulator promotes trust building activities and promotes a combination of functional trust and functional distrust approach.

The diverse risk profiles, competence level, and the legislative versus non-legislative element of the regimes were vital in understanding regulators approach to trust or distrust. High risk implied a strong regulation that gave the regulator a large range of choices in approaching the regulatees. In such a situation (health care and petroleum) reglators may vary their approach depending on if they trust in the regulatees. On the other hand a weak regulation (municipal emergency management) leaves the regulator with fewer options in practice. Persuasion, dialogue, and communicative approaches may work well when facing organizational incompetence. But such an approach would be dysfunctional if distrust characterizes the relation and the regulatee is perceived as an amoral calculator $[20,22,24,26]$. 


\section{References}

[1] U. Beck, Risk Society. Towards a New Modernity. New Dehli: Sage. 1991.

[2] B. Barber, The Logics and Limits of Trust. Rutgers University Press. 1983

[3] A. Giddens, Modernity and Self-Identity: Self and Society in the late Modern Age. Cambridge: Polity Press. 1991.

[4] N. Luhmann, Trust and power. Chicester, England; Wiley. 1979.

[5] R.J. Lewicki, E.C., Tomlinson, N., Gillespie, Models of Interpersonal Trust: Theoretical Approaches, Empirical Evidence, and Future Directions. Journal of Management, 32(6), (2006), pp. 991-1022.

[6] R. Seppänen, K. Blomqvist, S. Sundqvist, Measuring interorganizational trust - a critical review of the empirical research in 1990-2003. Industrial Marketing Management, 36, 2007, pp. 249-265.

[7] J. Child, G. Möllering,. Confidence and Active Trust Development in the Chinese Business Environment. Organization Science, 14 (2003), pp. 69-80.

[8] J.H. Dyer, W. Chu. The Role of Trustworthiness in Reducing Transaction Costs and Improving Performance: Empirical Evidence from the United States, Japan and Korea. Organization Science, 14 (2003), pp. 57-68.

[9] K.M. Andrews, B.L. Delahaye, Influences of knowledge process in organizational learning: The psychological filter. Journal of Management Studies, 37(2000), pp. 797-811.

[10] I. Nonaka, H. Takeuchi, The knowledge creating company. New York: Oxford University Press, 1995.

[11] J. Reason, Managing the Risk of Organizational Accidents. Ashgate, USA, 1997.

[12] S. Jeffcott, N. Pidgeon, J. Walls, Risk, Trust and Safety Culture in the U.K. Train Operating Companies. Risk Analysis, 26 (2006),pp. 1205-1121.

[13] C. Hood, H. Rothstein, \& R. Baldwin, The Government of Risk - Understanding Risk Regulation Regimes. Oxford, Oxford University Press. 2001.

[14] S.M. Conchie, C. Burns, Trust and Risk Communication in High-Risk Organizations: A Test of Principles from Social Risk Research. Risk Analysis, 28(2008), pp. 141-149.

[15] J.E. Tharaldsen, K.J. Mearns, K. Knudsen, A comparative study of safety climate on the Norwegian and Continental Shelves. Proceedings SPE international conference, HSE, Nice (France), April 2008.

[16] J.E. Tharaldsen, "In safety we trust". PhD thesis nr 121, University of Stavanger, Norway, 201.1

[17] R.C. Mayer, J.H. Davis, F.D. Schoorman, An integrative model of organizational trust. Academy of Management Review, Vol. 20, (1995),pp. 709-734.
[18] J.H. Sullivan, R.B. Peterson, N. Kameda, J. Shimada, The Relationship Between Conflict Resolution Approaches and Trust - A Cross Cultural Study. Academy of Management Journal, 24(1981), pp. 803-815.

[19] S. Zaheer, A. Zaheer, Trust across border. Journal of International Business Studies, 37(2006), pp. 21-29.

[20] K. Walshe, Regulating health care. A prescription for improvement? Glasgow, Open University Press. 2003.

[21] K. Hawkins, \& J.M. Thomas, (eds.). Enforcing Regulation. Hingham, Kluwer-Nijhoff Publishing. 1984.

[22] I. Ayres, and J. Braithwaite, Responsive RegulationTranscending the Deregulation Debate. New York, Oxford University Press. 1992.

[23] R. Baldwin, and P. Cave, (eds.), Law and Uncertainty. Risk and Legal Processes. London, Kluwer Law International. 1997.

[24] R.A. Kagan, and J.T. Scholz, The "Criminology of the Corporation" and Regulatory Enforcement Strategies. In Hawkins, K. \& Thomas, J.M. (eds.) Enforcing Regulation. Hingham, Kluwer-Nijhoff Publishing. 1984.

[25] T. Reiman, and L. Norros,. Regulatory Culture: Balancing the Different Demands of Regulatory Practice in The Nuclear Industry. In Kirwan, B., Hale, A \& Hopkins, A. (eds.) Changing Regulation - Controlling Risks in Society. Oxford, Pergamon. 2002.

[26] A. Reiss, Selecting strategies of social control over organizational life. In Hawkins, K. \& Thomas, J.M. (eds.). Enforcing Regulation. Hingham, Kluwer-Nijhoff Publishing. 1984.

[27] S. Wiig, Contributions to risk management in the public sector. PhD thesis nr 48, University of Stavanger, Norway, 2008.

[28] J.E. Tharaldsen, S. Wiig, B.A. Hanson, et al, (2011). Major accidents and their consequences for risk regulation. Accepted for the ESREL conference Troyes, France, 18.-22. September, 2011.

[29] Tharldsen, Mearns \& Knudsen, Perspectives on safety: the impact of group membership, work factors, and trust on safety performance in UK and Norwegian drilling companies, Safety Science, 48 (2010), pp. 1062-1072.

[30] M.Q. Patton, Enhancing the Quality and Credibility of Qualitative Analysis. Health Services Research, 34 (1999), pp. 1189-1208.

[31] S. Wiig, and K. Aase, K. (2007). Fallible humans in infallible systems? Learning from errors in health care. Safety Science Monitor, 11 (2007), Article 6.

[32] P.E. Hjort, Uheldige hendelser I helsetjenesten, Forslag til nasjonalt program for forebygging og håndtering, Sosial og Helsedirektoratet, 2003.

[33] H.S. Logstein, Myndighetenes forvaltning av sikkerhetsregelverket i petroleumsvirksomheten. 
[Authorities' management of safety regulations in the petroleum sector]. 2009.

[34] J.E. Karlsen, and P.H. Lindøe, The Nordic OHS model at a turning point? Policy and Practice in Health and Safety, 4 (2006), pp. 17-30.

[35] A. Bruhn, The inspector's dilemma under regulated selfregulation. Policy and Practice in Health and Safety, 4 (2006), pp.3-23.

[36] R.J. Lewicky, D.J. Mcallister, R.J. Bies, Trust and distrust: New relationships and realities. Academy of Mangement review, 23 (1998), pp. 438-458.
[37] T.K. Das, and B.S. Teng,. Between Trust and Control: Developing Confidence in Partner Cooperation in Alliances. Academy of Management Review, 23(1998), pp. 491-512.

[38] C. Argyris, The impact of budgets on people. New York: Controllership foundation, 1952.

[39] M. Goold, and A. Campbell, Strategies and styles: The role of the centre in managing diversified corporations. Oxford, England: Basil Blackwell, 1987. 